\title{
MAPEAMENTO DA PRODUÇÃO CIENTIFICA SOBRE PROTÓTIPOS NO DESENVOLVIMENTO DE PRODUTOS ELETROMÉDICOS
}

Thiago Bertolini dos Santos (thiagobertolinis@gmail.com) - Escola de engenharia de São Carlos Universidade de São Paulo (EESC - USP)

Lucas Portilho Camargos Gomes (lucaspcgomes@usp.br) - Escola de engenharia de São Carlos Universidade de São Paulo (EESC - USP)

Janaina Mascarenhas Hornos da Costa (janainamhcosta@gmail.com) - Escola de engenharia de São Carlos - Universidade de São Paulo (EESC - USP)

\section{RESUMO}

Prototipagem pode ser definida como um processo de concepção de uma representação de um produto final e é uma ferramenta essencial na avaliação da usabilidade. A usabilidade é definida como a facilidade do usuário em manusear o produto e suas funcionalidades. O protótipo é um meio interativo de representação visual de ideia e de comunicação entre os usuários e os desenvolvedores. A criação de protótipos é uma maneira rápida para identificar e incorporar o feedback do usuário no projeto. Muitas vezes o setor da saúde apresenta grandes falhas em produtos, devido à falta de consideração de requisitos de usabilidade importantes para os usuários. Isto posto, este trabalho tem como objetivo mapear as publicações que apresentam casos de aplicação de protótipos no setor da saúde detectando os autores, instituições, principais grupos de pesquisa e temas de maior interesse. Para isto, a metodologia adotada foi a combinação de uma revisão bibliográfica sistemática (RBS) $e$ análise bibliométrica. Primeiramente levantou-se os artigos através da revisão bibliográfica sistemática proposta por Conforto; Amaral; Silva, (2011). Então, foi feito a análise bibliométrica por meio dos softwares VantagePoint, UCINet e NetDraw. Através dessa metodologia percebeu-se que as publicações da área cresceram a partir do ano de 2003 e que a maioria da publicações e instituições com interesse nesse assunto estão localizadas nos Estados Unidos. Vale ressaltar que a análise mostrou uma dispersão muito grande entre os autores e que apenas Zhang,.J. possui 2 publicações selecionadas pelo protocolo da RBS efetuada, o que não possibilitou a identificação de grupos de pesquisas. Uma limitação dessa pesquisa parte da aplicação de apenas uma base de dados (SCOPUS) como fonte dos artigos.

Palavras-chave: protótipo, envolvimento do usuário, prototipagem, usabilidade.

Área: Ferramentas e métodos de desenvolvimento de produtos e serviços

\section{INTRODUÇÃO}

Para atingir um resultado com maior valor agregado para os stakeholders, os projetos de novos produtos devem ser avaliados ao longo do Processo de Desenvolvimento de Produtos (PDP). Essa avaliação propicia tornar o produto bem idealizado. O produto deve ser eficaz, seguro e atender as necessidades das pessoas que irão utilizá-lo. Isto exige a consideração de um número de fatores, incluindo os recursos e padrões de trabalho, as necessidades e estilos de vida dos usuários, os ambientes em que vão ser utilizado e o sistema de que ele irá fazer parte (MARTIN et al., 2012).

Abras; Maloney-krichmar; Preece, (2004) destacam a necessidade de envolver os usuários no projeto de desenvolvimento e afirmam que isto se tornou parte central do processo de 
desenvolvimento, pois esse envolvimento resulta em produtos mais eficazes, eficientes e mais seguros e contribui para a aceitação e sucesso do produto.

Nesse cenário Gulliksen et al., (2003) ressaltam a importância do "User-Centred Design" (UCD), como um processo que foca a usabilidade ao longo de todo o processo de desenvolvimento e também ao longo do ciclo de vida do sistema/produto. A usabilidade revela o nível de eficiência e eficácia em que o usuário atinge o objetivo do produto, com relação a meta proposta por um produto, em uma determinada situação de uso (IVORY; HEARST, 2001). Uma ferramenta essencial na avaliação da usabilidade é a prototipagem. Ela fornece uma representação mais próxima das características do produto final e é utilizado para identificar e incorporar o feedback dos stakeholders em um projeto.

Os protótipos podem ser de vários materiais e aspectos, incluindo amplitude de recursos (característica), grau de funcionalidade, grau de interação, e refinamento estético (VIRZI; SOKOLOV; KARIS, 1996). Além disso, diferentes tipos de protótipos podem ser construídos ao longo do projeto, incorporando diferentes níveis de detalhes, uma vez que cada tipo de protótipo se destaca em avaliar diferentes variáveis do projeto ligadas ao usuário (VIRZI; SOKOLOV; KARIS, 1996).

A prototipagem possui vários propósitos, como: fomentar a comunicação, mediar prioridades de requisitos, avaliar funcionalidade, avaliar usabilidade entre outros. Segundo Rinkus et al., (2005) a cultura do setor da saúde ainda é de treinar as pessoas para se adaptarem ao sistema produto ao invés de projetar este para atender as necessidades do usuário. Assim, o "UserCentred Design" (UCD), como descrito acima não é aplicado, resultando em projetos falhos pela falta de consideração dos requisitos importantes para o usuário.

Para Han; Shin; Chow, (2016) particularmente no setor da saúde existe uma variação de usuários como pacientes, cuidadores e médicos. Isso dificulta generalizar os requisitos de concepção do sistema/produto, assim, as necessidades do cuidador possuem um foco maior na eficiência do tratamento enquanto a do paciente foca no conforto que o sistema/produto proporciona. Com as falhas ocasionadas por incoerências no design, Martin et al., (2012) ressalta que os padrões de usabilidade passam a ser fatores importantes para demonstrar conformidade para os produtos médicos.

Considerando a importância de elaborar protótipos no desenvolvimento de um produto e sua efetividade na concepção de um produto que atenda as necessidades dos usuários, a identificação das teorias já existentes sobre o assunto é importante para fundamentar futuras pesquisas. Assim, propusemos a seguinte questão de pesquisa: Qual nível de conhecimento ou de desenvolvimento no estudo de protótipos no setor de produtos eletromédicos? Com objetivo de responder esta questão esta pesquisa busca mapear os principais autores, grupos de pesquisa, instituições e temas de interesse.

\section{MÉTODO DE PESQUISA}

Nessa pesquisa usou-se como metodologia a combinação de uma revisão bibliográfica sistemática (RBS) e análise bibliométrica. A RBS foi realizada baseada na RBS roadmap proposta por Conforto; Amaral; Silva, (2011), que é recomendada para pesquisas na área de gestão de operações, com foco em desenvolvimento de produtos e gestão de projetos e está organizada em 15 etapas e divididas em 3 fases (entrada, processamento e saída).

\section{$2.11^{\text {a }}$ Fase - Entrada}

Essa fase, é onde ocorre a definição do problema, objetivo, fontes primárias, strings de busca, critérios de inclusão, critérios de qualificação, métodos e ferramentas e cronograma. Dessa 
maneira, propicia o ponto de partida da revisão bibliográfica. Para esta pesquisa, no entanto, será detalhado apenas as etapas de objetivo, fontes primárias, string de busca e critérios de inclusão, os quais são os mais importantes para uma análise bibliométrica.

O objetivo desta revisão bibliográfica, foi encontrar os propósitos dos protótipos em equipamentos eletromédicos. Assim, primeiramente foram levantados alguns artigos, os quais foram considerados como estado da arte do tema protótipos e que pudessem contribuir para o conjunto de termos utilizados nessa área. Isto posto, algumas palavras como UCD, User Centered Design, User Centred Design e Prototype foram identificadas. Com base nessas fontes e nos termos identificados a seguinte string de busca foi gerada.

Quadro 1 - String de busca

(TITLE-ABS-KEY ( ucd ) OR TITLE-ABS KEY ( user AND centered AND design ) OR TITLE-ABS KEY ( user AND centred AND design ) ) AND TITLE-ABS KEY ( prototype ) AND ( LIMITTO ( LANGUAGE, "English" ) ) AND ( LIMIT-TO ( SRCTYPE, "j" ))

Fonte - Produzido pelos autores

Para atingir o objetivo da RBS os critérios de inclusão são importantes na análise do resultado da fase de processamento. Esses critérios serão responsáveis pelo refinamento dos resultados e terão um impacto considerável na análise bibliométrica. Dessa forma, o critério de inclusão dessa pesquisa foi apresentar uma "Proposição de prototipagem para produtos médicos"

\section{2 $2^{\mathrm{a}}$ Fase - Processamento}

Em uma primeira iteração da RBS roadmap executando a string de busca do quadro acima atingimos o resultado de 339 artigos. Essa busca foi realizada em trabalhos escritos com a língua inglesa e na base SCOPUS. Posteriormente foram lidos os resumos desses artigos e aplicado o fator de inclusão: proposição de prototipagem para produtos médicos. Assim, gerou-se um seleto grupo de 62 artigos, dos quais teve-se acesso a 53 deles.

\section{$2.33^{\text {a }}$ Fase - Saída (Análise bibliométrica)}

A saída é a etapa da análise bibliométrica. Essa foi realizada com os softwares VantagePoint, UciNet e NetDraw. O Vantage point, foi o responsável por coletar os dados (publicações, instituições, autores, palavras chaves) dos 53 artigos selecionados e trata-los em forma de matrizes e contagens. O UciNet é um software especializado em análise dos dados, assim, os dados de saída de VantagePoint se tornaram inputs para o UciNet, que propiciou a análise das redes. Por fim, o Netdraw foi utilizado para gerar e visualizar as redes.

\section{RESULTADOS}

Os resultados encontrados na análise bibliométrica são apresentados e discutidos a seguir.

\subsection{Publicações}

Conforme mostrado na figura 1, dentro da RBS em questão, percebe-se que a quantidade de publicações relacionadas com prototipagem na área médica antes de 2003 era muito pequena. A partir desta data, houve uma evolução considerável, mas sempre tendo oscilações. O ápice de quantidade de publicações foi atingido em 2016 tendo como resultado a publicação de 9 documentos. Isso mostra que o assunto está ganhando atenção nos últimos anos. 
Figura 1 - Quantidade de publicações por ano

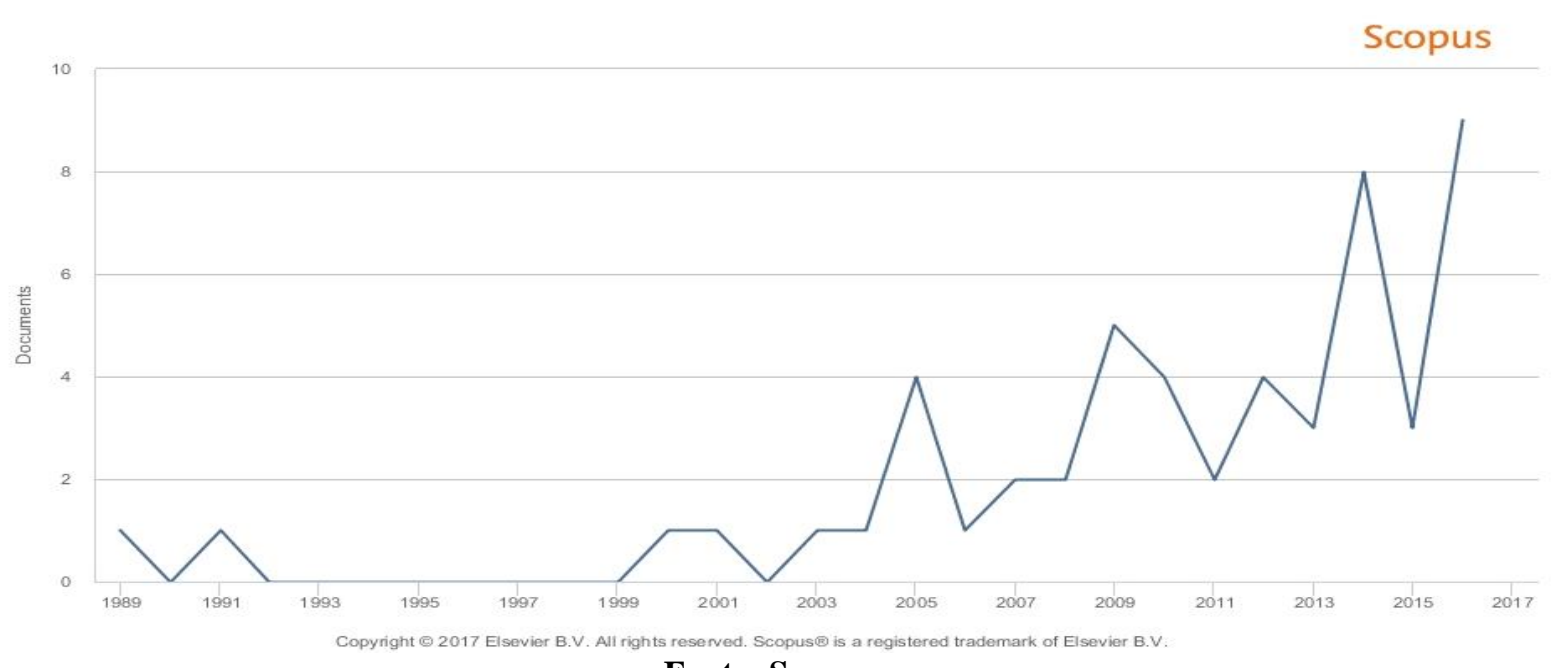

Fonte: Scopus

\subsection{Instituições}

O assunto prototipagem de produtos no setor médico é bastante disperso em relação as instituições. O quadro 2 apresenta os Estados unidos como o pais que se destaca em quantidade de publicações na área, tendo participado de 22 dos 53. No entanto, as quantidades são bem dividias entre as instituições que estão localizadas nesse território, não tendo uma se sobressaindo sobre as outras. É possível verificar no quadro 3 que as publicações são bastante segmentadas entre as instituições. Isso, possibilita concluir que ainda não há uma instituição que desponta no assunto, contudo, o interesse é existente em um número considerável de instituições.

Quadro 2 - Quantidade de publicações por países

\begin{tabular}{|c|c|}
\hline Quantidade de publicações & País da publicação \\
\hline 22 & Estados Unidos \\
\hline 7 & Reino unido \\
\hline 4 & Holanda \\
\hline 3 & Austrália \\
\hline 3 & Canada \\
\hline
\end{tabular}

Fonte - Produzido pelos autores

Quadro 3 - Quantidade de publicações por Instituições

\begin{tabular}{|c|c|}
\hline Quantidade de publicações & Instituição da publicação \\
\hline 2 & University of Central Florida, Orlando \\
\hline 2 & University of Utah, Salt Lake City \\
\hline 2 & University of Virginia, Charlottesville \\
\hline 2 & Vanderbilt University, Nashville \\
\hline
\end{tabular}

Fonte - Produzido pelos autores

\subsection{Autores}

Conforme mencionado na seção acima, este é um assunto bastante disperso ainda na literatura. Portanto, não encontramos um autor que se destacasse no tema. A análise bibliométrica mostrou, de acordo com a figura 2, uma única recorrência de autores em escrever sobre o assunto. Assim, identificamos 228 autores envolvidos em 53 publicações e 
apenas o Zhang, J. possui 2 publicações dentro da RBS, os demais todos possuem apenas 1 publicação cada.

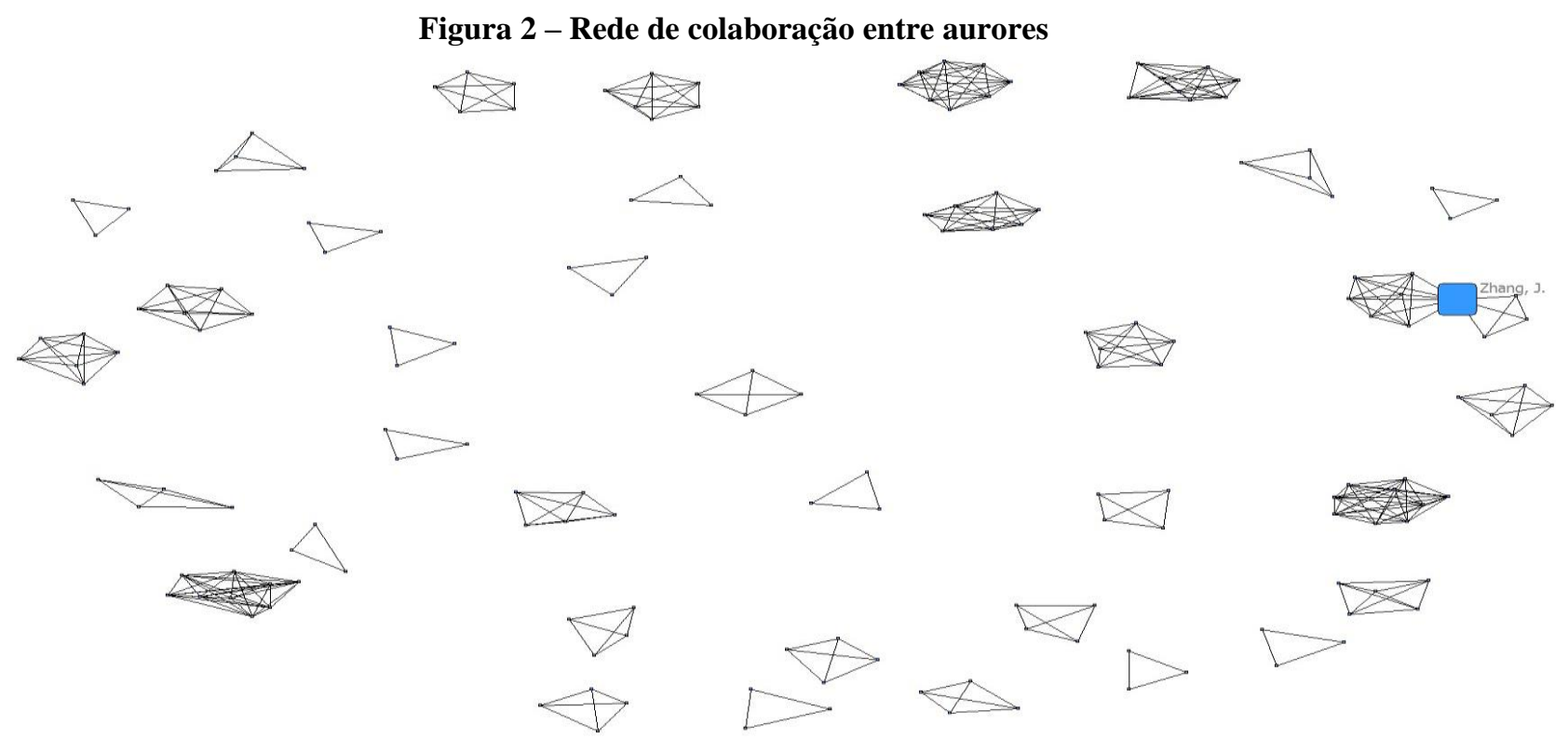

Fonte - Produzida pelos autores

Dessa forma, não é possível afirmar que existem grupos de pesquisas bem definidos. Com a rede de colaboração entre autores ou rede de co-autoria, verificamos que apenas o Zhang, J. media 2 grupos diferentes de pesquisa, isto é, participa de 2 publicações diferentes.

\subsection{Palavra-chave}

Nessa análise identificou-se os temas mais utilizados nesse assunto. Nos 53 artigos referentes a prototipagem em produtos médicos foram encontradas 175 palavras-chave diferentes. Isso mostra uma heterogeneidade muito grande entre os termos usados. Das 175 palavras-chave 159 aparecem apenas 1 vez, 12 delas aparecem 2 vezes e outras 3 aparecem 4 vezes nos artigos da RBS. O destaque é para o termo User centered design que aparece 16 vezes (vide tabela abaixo que apresenta as 16 palavras mais utilizadas).

Tabela 1 - Frequência de palavras-chaves

\begin{tabular}{|cc|cc|}
\hline Frequência & Palavra-chave & Frequência & Palavra-chave \\
\hline 16 & User centered design & 2 & Glaucoma \\
4 & Prototype & 2 & Medication management \\
4 & Usability & 2 & Participatory design \\
4 & User interface & 2 & Patient-centered care \\
2 & Assistive Technologies & 2 & Requirements elicitation \\
2 & Clinical decision support system & 2 & User experience \\
2 & Collaborative design & Healthcare \\
2 & Communication & & Outras \\
2 & Design &
\end{tabular}

No entanto, entre os temas denominados como "outros", existem algumas palavras que podem ser consideradas como expressões com sentido aproximado. E dependendo da análise podem se tornar parte de um grupo em comum. Como é o caso das tabelas abaixo: 
Tabela 2 - Expressões similares

\begin{tabular}{|c|c|c|c|}
\hline \multicolumn{2}{|c|}{ Similaridade com prototype } & \multicolumn{2}{|c|}{ Similaridade com Usability } \\
\hline Frequência & Palavra-chave & Frequência & Palavra-chave \\
\hline 1 & Computer-based prototyping & 1 & Usability analysis \\
\hline 1 & Digital prototyping & 1 & Usability heuristics \\
\hline 1 & Paper prototype & 1 & Usability testing \\
\hline 1 & Paper prototyping & & \\
\hline 1 & Prototype implementation & & \\
\hline 1 & Prototyping & & \\
\hline 1 & rapid prototyping & & \\
\hline 1 & Tactile paper prototyping & & \\
\hline 1 & Virtual prototype & & \\
\hline 1 & Virtual-mixed prototyping & & \\
\hline
\end{tabular}

A tabela 2, expõe as quantidades de expressões similares que são utilizadas na área. Sendo assim, se concentrássemos, por exemplo, todas as expressões similares de protótipos na palavra-chave prototype, teríamos uma frequência maior da mesma, que passaria de 4 para 14 .

Percebe-se que existe uma variação muito grande de combinações de palavras-chave para essa área. Assim sendo, na figura 3 verificou-se que as palavras chaves se combinam poucas vezes do mesmo modo, isto é apenas a palavra User Centered Design combina mais de 1 vez com as palavras glaucoma e prototype.

Entre esses temas mencionados acima (tabela 1), existem intermediações, isto é, possuem palavras que aparecem em conjunto para identificar a característica do conteúdo global. Também é visível (figura 3) que a tríade responsável por essa função é composta pelas palavras User Centered Design, Usability e Prototype (pontos azuis maiores). Essas palavras mediam o assunto nos diferentes aspectos que ele pode percorrer, isso quer dizer que na maioria das vezes pelo menos uma delas ou suas similares, estarão nas combinações efetuadas em RBSs desse conteúdo.

Figura 3 - Redes de palavras-chave

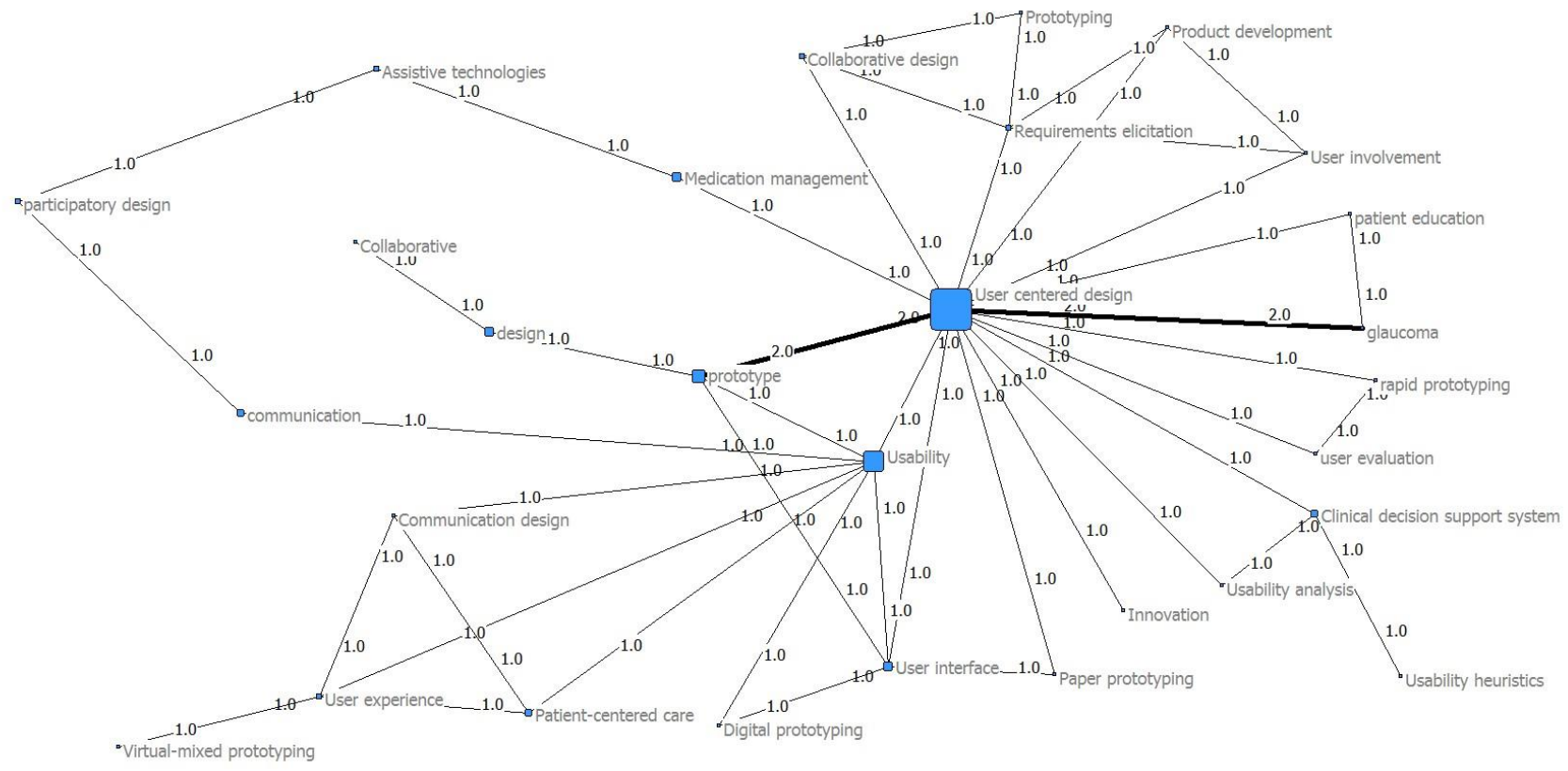

Fonte - Produzida pelos autores 


\subsection{REFERÊNCIAS}

A análise bibliométrica revela que para essa área de prototipagem em produtos médicos não existe um ou mais trabalhos que se destaquem e sejam citado frequentemente. Conforme tabela abaixo existe algumas pesquisas que se sobressaem, no entanto, de forma sutil.

Quadro 4 - Quantidades de citações por referências

\begin{tabular}{|c|c|}
\hline $\begin{array}{c}\text { Quantidades } \\
\text { de citações }\end{array}$ & Referências \\
\hline 6 & NIELSEN, Jakob. Usability engineering. Elsevier, 1994. \\
\hline 4 & $\begin{array}{r}\text { INTERNATIONAL ORGANIZATION FOR STANDARDIZATION. Human-centred Design } \\
\text { Processes for Interactive Systems. International Organization for Standardization, } 1999 .\end{array}$ \\
\hline 4 & PREECE, J. Rogers et al. s. Carey, T. (1994). Human-computer interaction \\
\hline 3 & BEYER, Hugh; HOLTZBLATT, Karen. Contextual design: defining customer-centered \\
systems. Elsevier, 1997.
\end{tabular}

\section{Fonte - Produzida pelos autores}

Essas pesquisas, são citadas nos artigos coletados através da RBS, mas aparentemente não existe um senso comum sobre um trabalho referência de estado da arte para a área. É compreensível, de acordo com a figura 4 e vide quadro 4, que as 3 referências mais citadas (pontos vermelhos maiores) alimentam o trabalho Integrating user centered design, universal design and goal, operation, method and selection rules to improve the usability of DAISY player for persons with visual impairments, o qual foi extraído da RBS (ponto azul). Assim, percebe-se que o conjunto das referências mais citadas prevalecem em alguns trabalhos. Isso pode mostrar o surgimento de um conjunto de trabalhos base para a área.

Figura 4 - Rede de citação

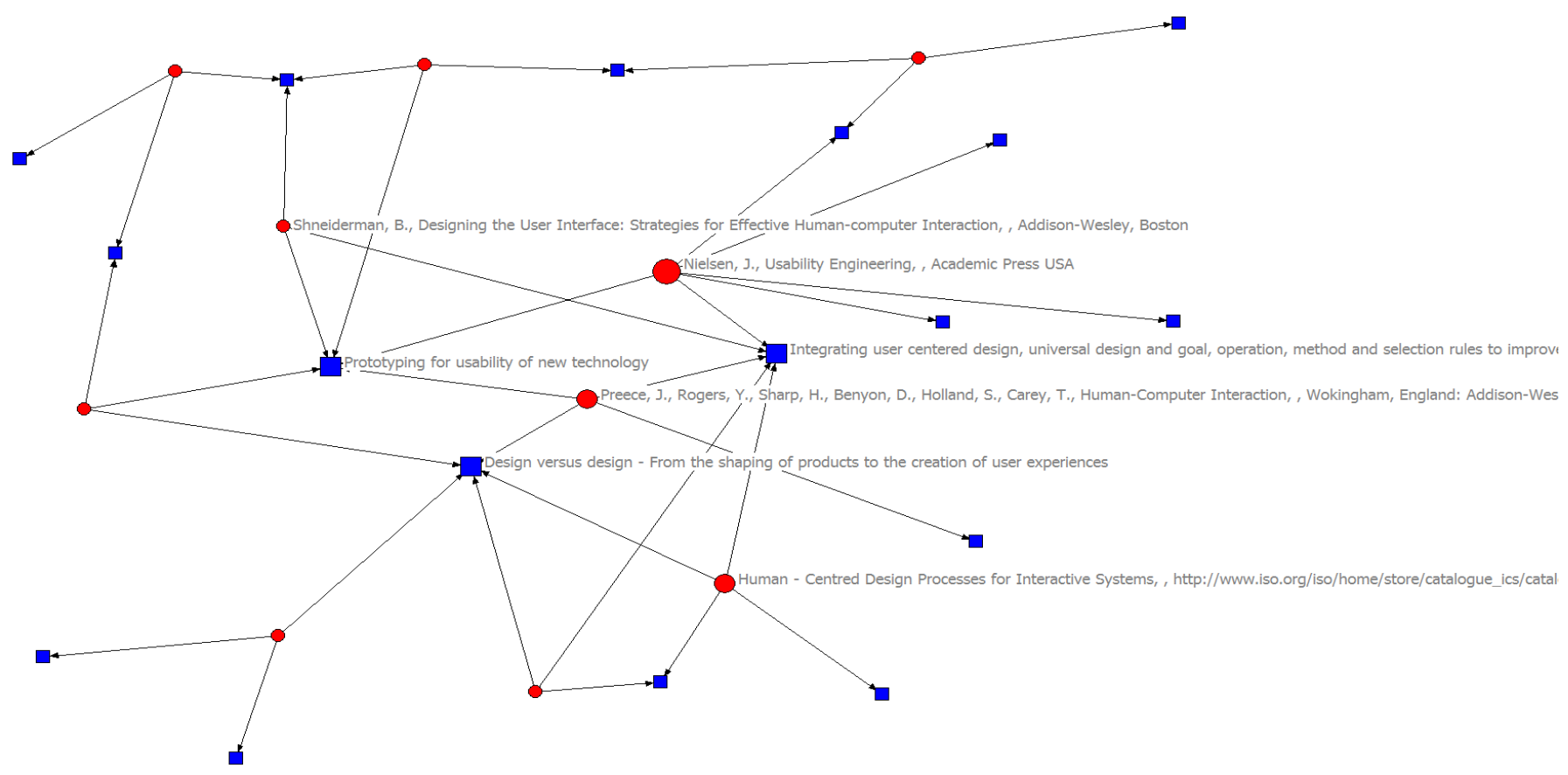

Legenda - Pontos azuis $=$ Trabalhos extraídos da RBS; Pontos Vermelhos $=$ Referências

Fonte - Produzida pelos autores 
Apesar de não ter trabalhos com alta recorrência, alguns autores se sobressaem por terem uma quantidade alta de trabalhos sendo citados. Isso demonstra que a base dos conceitos é retirada de alguns autores já consagrados, no entanto, estes não entraram com veemência na área especifica (prototipagem em produtos médicos). Estes autores estão descritos na tabela abaixo:

Tabela 3 - Quantidade de citação por autor

\begin{tabular}{|cc|cc|cc|}
\hline $\begin{array}{c}\text { Quantidade } \\
\text { de citação }\end{array}$ & Autor & $\begin{array}{c}\text { Quantidade } \\
\text { de citação }\end{array}$ & Autor & $\begin{array}{c}\text { Quantidade } \\
\text { de citação }\end{array}$ & Autor \\
\hline 19 & Nielsen J & 7 & Holtzblatt K & 6 & Virzi RA \\
9 & Norman DA & 7 & Preece J & 6 & Ehn P \\
9 & Zhang J & 6 & Grudin J & 6 & Hanna GB \\
8 & Rolland JP & 6 & Staggers N & 5 & Beyer H \\
8 & Sharp H & 6 & Shneiderman B & 5 & Elwyn G \\
8 & Rogers Y & 6 & Oinas-Kukkonen H & 5 & \\
8 & Kujala S & 6 & Patel VL & &
\end{tabular}

\section{CONSIDERAÇÕES FINAIS}

Este trabalho teve como objetivo mapear a produção cientifica de protótipos no desenvolvimento de produtos eletromédicos. Para isso, realizou-se uma RBS roadmap proposto por Conforto; Amaral; Silva, (2011) e em sequência uma análise bibliométrica.

A princípio, através das palavras-chave, foram identificados 339 artigos, os quais foram refinados e selecionados 62 artigos através do critério de inclusão: proposição de prototipagem para produtos médicos. Por fim, obteve-se acesso a 53 artigos, para a análise bibliométrica através dos softwares Vantage Point, UciNet e Net Dreaw.

Os resultados mostraram que o número de publicações na área vem crescendo a partir do ano de 2003. Essas publicações se destacam em quantidade nos Estados Unidos, porém, não existe uma instituição com maior evidencia até o momento. Isso revela uma dispersão considerável sobre o tema.

Por se tratar de um assunto disperso não encontrou-se um trabalho de destaque na área, nem mesmo um grupo de pesquisa relevante. Na RBS realizada, apenas o autor Zhang, J. teve 2 publicações diferentes, os demais tiveram somente uma. As referências que se sobressaíram de forma sutil, foram algumas de autores já consagrados, contudo, que não tratam o assunto até este momento com veemência. Percebe-se isto, através do número considerável de citações que estes autores tiveram (tabela 3), mas nenhum destes trabalhos se destacou.

Com a utilização de 175 palavras-chave diferentes, percebe-se também uma grande heterogeneidade de palavras-chave utilizadas na área. Isso demonstra que ainda o conhecimento está no início de sua construção. Ainda assim, podemos perceber o início de um grupo de palavras de destaque, isto é, que intermedeiam todas as outras, são elas: User Centered Design, Usability e Prototype.

Por fim, podemos concluir que o conhecimento ainda está no início de sua construção e com algum interesse de muitos autores. Os conceitos bases do assunto são referenciados de autores consagrados para produtos em geral, mas o interesse concentrado na área médica vem crescendo e as adaptações focadas nessa área vem acontecendo. Dessa forma, é importante ressaltar que é uma área promissora e de grande potencial para futuras pesquisas, visto que, é essencial que os produtos médicos tenham usabilidades e atendam as necessidades de seus usuários. 


\section{REFERÊNCIAS}

ABRAS, C.; MALONEY-KRICHMAR, D.; PREECE, J. User-Centered Design, 2004. (Nota técnica).

CONFORTO, E. C.; AMARAL, D. C.; SILVA, S. L. DA. Roteiro para revisão bibliográfica sistemática: aplicação no desenvolvimento de produtos e gerenciamento de projetos. $8^{\circ}$ Congresso Brasileiro de Gestão de Desenvolviemnto de Produto - CNGDP 2011. Anais...2011Disponível em: 〈http://www.ufrgs.br/cbgdp2011/downloads/9149.pdf>

GULLIKSEN, J. et al. Key principles for user-centred systems design. Behaviour \& Information Technology, v. 22, n. 6, p. 397-409, 2003.

HAN, F.; SHIN, K.; CHOW, D. User-centred design approach for hydrotherapy wetsuit. International Journal of Fashion Design, Technology and Education, v. 9, n. 1, p. 16-22, 2016.

IVORY, M. Y.; HEARST, M. A. The state of the art in automating usability evaluation of user interfaces. ACM Computing Surveys, v. 33, n. 4, p. 470-516, 2001.

MARTIN, J. L. et al. A user-centred approach to requirements elicitation in medical device development: A case study from an industry perspective. Applied Ergonomics, v. 43, n. 1, p. 184-190, 2012.

RINKUS, S. et al. Human-centered design of a distributed knowledge management system. Journal of Biomedical Informatics, v. 38, n. 1, p. 4-17, 2005.

VIRZI, R.; SOKOLOV, J. L.; KARIS, D. Usability problem identification using both lowand high-fidelity prototypes. Proceedings of the SIGCHI conference on Human factors in computing systems common ground, p. 236-243, 1996. 